\title{
A systematic review of interventions to increase attendance at health and fitness venues: identifying key behaviour change techniques
}

\author{
Matthew Rand ${ }^{1 *}$, Paul Norman ${ }^{2}$ and Elizabeth Goyder ${ }^{1}$
}

\begin{abstract}
Background: Members' attendance at health and fitness venues typically declines over the course of their membership, with a likely negative impact on physical activity and health outcomes. This systematic review sought to examine the effectiveness of interventions to increase attendance at health and fitness venues and identify the behaviour change techniques (BCTs) included in effective interventions.

Methods: A systematic search of seven databases was conducted. The Behaviour Change Technique Taxonomy was used to code the interventions. Cohen's $d$ was used to assess the effectiveness of the interventions.

Results: Fourteen papers reporting 20 interventions were included in the review. Most interventions were found to have trivial or small effects on attendance, although one had a medium effect $(d=0.60)$ and three had a large effect $(d s=1.00$, $1.37,1.45$ ). The interventions used a limited range of $B C T s$, with "Prompts/Cues" being the most frequently used. Of the interventions with large effect sizes, two used "Problem solving" and "Pros and cons" and one used "Goal setting (behaviour)" and "Review behaviour goals".

Conclusions: Only a small number of studies have tested interventions to increase attendance at health and fitness venues, with predominantly trivial or small effects. With the possible exception of problem solving alongside decisional balance and goal setting alongside reviewing behaviour goals, there is little evidence for the effectiveness of specific BCTs. Further research is required to identify the key components of effective interventions to increase attendance at health and fitness venues.
\end{abstract}

Keywords: Physical activity, Public health, Health and fitness, Interventions, Attendance, Behaviour change

\section{Background}

Worldwide, it is estimated that $31 \%$ of adults aged 15 and over are inactive; that is, they do not meet the recommended guidance of $150 \mathrm{~min}$ of moderate-intensity aerobic physical activity (PA), or at least $75 \mathrm{~min}$ of vigorous-intensity aerobic PA, per week [1]. Thus, there

\footnotetext{
* Correspondence: mrand1@sheffield.ac.uk

'School of Health and Related Research, The University of Sheffield, 30 Regent St, Sheffield S1 4DA, UK

Full list of author information is available at the end of the article
}

is a clear need to increase PA in a significant proportion of the population. Public Health England (PHE) has identified a range of sectors that are well positioned to help the population become more active including local and national government, schools, health services, the transport sector, voluntary organisations and the sport and leisure sector [2]. Of these, the sport and leisure sector is the only one to provide PA as a direct service and is therefore well placed to support increases in PA levels. However, to date, there is limited evidence about how

(c) The Author(s). 2020 Open Access This article is licensed under a Creative Commons Attribution 4.0 International License, which permits use, sharing, adaptation, distribution and reproduction in any medium or format, as long as you give appropriate credit to the original author(s) and the source, provide a link to the Creative Commons licence, and indicate if changes were made. The images or other third party material in this article are included in the article's Creative Commons licence, unless indicated otherwise in a credit line to the material. If material is not included in the article's Creative Commons licence and your intended use is not permitted by statutory regulation or exceeds the permitted use, you will need to obtain permission directly from the copyright holder. To view a copy of this licence, visit http://creativecommons.org/licenses/by/4.0/ The Creative Commons Public Domain Dedication waiver (http://creativecommons.org/publicdomain/zero/1.0/) applies to the data made available in this article, unless otherwise stated in a credit line to the data. 
this sector can increase PA levels in the population [3]. Health and fitness is a large subsector of the sport and leisure industry, with approximately 60 million people in Europe having membership of a health and fitness organisation which gives them access to a venue [4]. Within the UK, approximately $15 \%$ of the population are estimated to be members of a health and fitness organisation [5]. Health and fitness venues typically provide PA equipment that can be used within gyms, they offer exercise classes led by trained instructors and offer swimming pool provision. Individuals typically pay a membership fee to use these facilities. Given that health and fitness organisations provide venues and activities that have the potential to increase PA levels in the population, and that many individuals primarily subscribe to use health and fitness facilities for health reasons (e.g., to lose weight, for increased fitness) [6], they provide an ideal context in which to study initiatives to increase PA levels.

Despite the level of health and fitness membership, attendances at health and fitness venues generally decline from the start of an individuals' membership [7]. Moreover, many members do not use their membership [8]. A recent study in the UK found that only $22 \%$ of new members attended a health and fitness venue 12 months after the start of their membership [7]. A study in the United States also reported a mean attendance of approximately four times a year for members on an annual contract [8]. It is likely that many of these members are not meeting recommended PA guidelines, given that most members join for health reasons [6]. Therefore, interventions that increase attendance at health and fitness venues are also likely to have a positive impact on public health.

To identify the most effective interventions to increase attendances at health and fitness venues, it is important to understand which interventions have previously been tried, the extent to which they have influenced attendance behaviour, and the intervention components that were key to behaviour change. Such research can provide useful information for health and fitness organisations about where to place their resources to increase member attendances at their venues. Such information would also be useful for national policy makers and global organisations such as the WHO to help inform future recommendations for promoting PA [9] (e.g., 'What Works' guidance). To date, very little is known about the effectiveness of interventions to increase attendance at health and fitness venues; the current review aims to fill this gap.

Coding a behaviour change technique (BCT), defined as an observable and replicable component of an intervention designed to alter processes that regulate behaviour within an intervention [10], can help to identify the key techniques, or "active ingredients", of an intervention. Understanding interventions that are effective in promoting behaviour change requires clear reporting and a standard for outlining the content and descriptions of interventions [11]. Thus, the current review utilised the $93 \mathrm{BCT}$ taxonomy (v1) [10] to code interventions that have attempted to increase attendances at health and fitness venues. Effective BCTs have been identified for promoting PA in general [12]. However, to date, there has been no research investigating the $\mathrm{BCT}$ s used in interventions aimed to increase attendance at health and fitness venues. The BCTs that help to increase PA may or may not be the same as those that are important in increasing attendance at health and fitness venues.

This systematic review therefore aimed to: 1) assess the effectiveness of interventions designed to increase attendance at health and fitness venues; 2) identify the $\mathrm{BCT}$ s that have been used in interventions to increase attendance at health and fitness venues; and 3) assess the relative effectiveness of different $\mathrm{BCTs}$ used to increase attendance at health and fitness venues.

\section{Method}

Search strategy, selection criteria and data extraction

Relevant health, psychological and exercise related electronic databases were selected; Business Source Premier, Cochrane Controlled Trials Register, Google Scholar, MEDLINE, Physical Education Index, PsychINFO and Scopus. Searches were carried out in June 2019. Only English language reports were included. There was no restriction on publication date. Reference lists and citations of identified studies were also scanned. Grey literature, including conference proceedings and abstracts were searched to identify research that may have been presented ahead of full publication. Only studies that tested interventions to increase attendance behaviour in a health and fitness venue using a randomised controlled experimental design were included in the review. Randomised controlled trials (RCTs) are considered to be the 'gold standard' design to provide evidence of effectiveness of an intervention and minimise the risk of bias [13]. Other designs such as nonrandomised or observational studies were excluded. Studies located in a health and fitness venue with adult members of the venue were included. Studies involving non-members or volunteers were excluded, as were studies involving participants who were suffering with a clinical condition or were part of an exercise referral scheme. Studies which only measured attendance at specific exercise sessions or programmes were excluded. The first author retrieved data, which was checked by the second author, from the included studies and recorded these on a standardised data extraction form. The following details were retrieved: author and country; sample; setting; conditions; BCTs; 
attendance measure; main findings; and effect size as assessed by Cohen's $d$ [14]. In line with Cohen's guidelines [14], $d<0.20$ was interpreted as trivial, $d \geq 0.20$ was interpreted as a small effect size, $d \geq 0.50$ as a medium effect size, and $d \geq 0.80$ as a large effect size. The conditions were coded such that a positive effect size would indicate a positive effect of the intervention on attendance relative to the control condition.

\section{Quality of the included studies}

The Cochrane tool to assess risk of bias in RCTs [15] was used to assess the quality of the included studies. This considers bias in terms of selection, performance, detection, attrition, reporting and other biases and studies are rated as high, low or unclear in the risk of bias for each domain. These criteria were used to rate each of the included studies.

\section{Behaviour change techniques (BCTs)}

The BCTs used in each study were identified from intervention descriptions and coded from the $\mathrm{BCT}$ taxonomy (v1) according to the instructions provided. BCTs in the intervention condition were coded. Where it was not possible to code an intervention component to one of the $93 \mathrm{BCTs}$ as described in the taxonomy, additional techniques were coded and named as appropriate.

\section{Results}

\section{Included studies}

Fourteen studies, including 20 interventions, were identified that met the inclusion criteria (see Fig. 1). The publication dates ranged from 1977 [16] to 2018 [17, 18]. A description of the included studies is presented in Table 1.

\section{Participants}

A total of 6788 participants were included in the 14 studies, with 3406 randomised to receive an intervention and 3382 to a control condition. The number of participants completing the studies (intervention and control) ranged from 36 [29] to 2463 [17]. The mean age of the participants ranged from 28 [29] to 41 [19] years old, with half of the studies reporting a mean age within the thirties. Twelve of the 14 studies included both females and males in their interventions. The remaining two studies only included females [16, 29]. Other demographics such as education, ethnicity and employment status were inconsistently reported.

\section{Mode of delivery}

The mode of delivery varied in the studies. There were two main modes of delivery; one study included multiple meetings with participants and was primarily face-toface [19], the remaining studies used methods which were not face-to-face (e.g., email reminders, telephone calls, letters).

\section{Length of intervention and monitoring periods}

Seven interventions were one-off interventions (e.g., a letter in the post) $[16,22,23,25,26,28,29]$ and the remaining seven interventions [17-21, 24, 27] varied between two weeks [18] and two years [19] in length. The median length was 12 weeks. All of the studies measured attendance either during the intervention and/or for a period of time after the intervention had taken place. The monitoring period of attendance ranged from two weeks [18] to two years [20]. The median monitoring period was 8 weeks.

\section{Outcome measures}

All 14 studies reported objective, electronically recorded attendance at the health and fitness venue.

\section{Quality of the included studies}

Overall, the included studies reported a low level of bias within the assessment. Bias was reported to be high once each in "random sequence generation" [20], "allocation concealment" [20], "selective reporting" [20] and "incomplete outcome data" [24]. A summary analysis of the level of bias in each of the included studies is presented in Fig. 2 and Table 2.

\section{Effectiveness}

Since it was not appropriate to combine the results of the included studies into a meta-analysis due to the heterogeneity of the interventions, exploratory analyses were performed to assess the effects of each of the interventions. Effect sizes were calculated to analyse which of the interventions had the largest effect on attendance over the control group and are reported in Table 1. Only two studies, reporting four interventions, reported a large $(d=1.00, d=1.37, d=1.45)$ or medium effect size $(d=0.60)[16,19]$. All of the remaining studies reported small or trivial effect sizes. Of the remaining studies, the largest was an effect size of $d=0.38$ [22] and the smallest was $d=0.004$ [27].

\section{Behaviour change techniques}

None of the studies explicitly reported the BCTs included in the interventions. Each intervention was therefore coded to identify BCTs in line with the BCT taxonomy (v1). Overall, 13 BCTs were coded across the 20 interventions. Four interventions included "Prompts/ cues" (BCT 7.1) [17, 18, 20, 23]). Three studies reported "Incentive (outcome)" (BCT 10.8) [21, 22, 27]). "Pros and cons" (BCT 9.2) ([16, 26]) was reported by two studies. Each of the following BCTs were reported once: "Goal setting (behaviour)" (BCT 1.1) [19], "Problem 


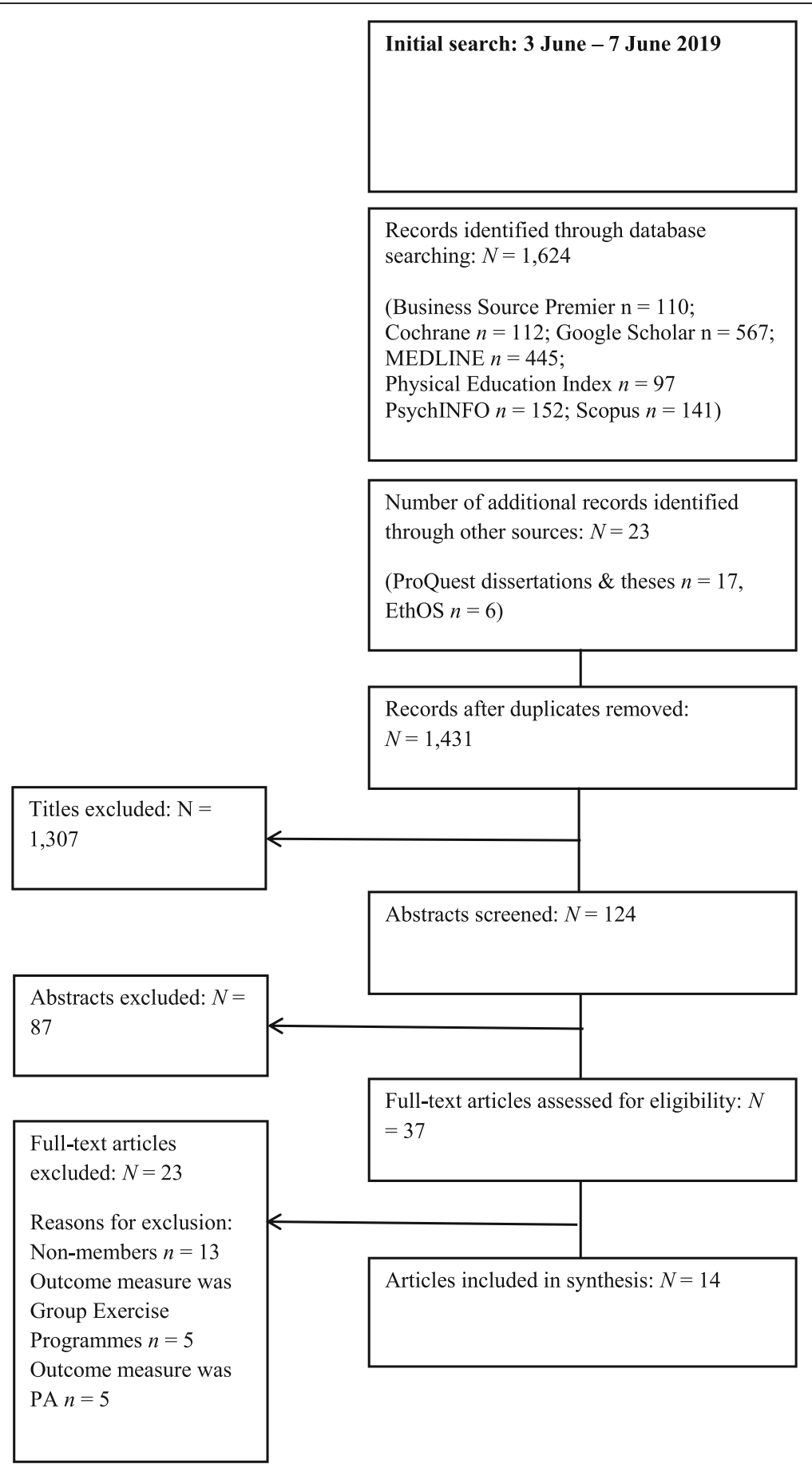

Fig. 1 PRISMA Flow Diagram Showing Study Selection Process

solving” (BCT 1.2), [16], “Action planning” (BCT 1.4) [18] "Review behaviour goal(s)" (BCT 1.5) [19], "Feedback on behaviour" (BCT 2.2) [27], "Self-monitoring of behaviour" (BCT 2.3) [25], "Material incentive (behaviour)" (BCT 10.1) [24] and "Future punishment" (BCT 10.11) [24]. Two additional BCTs were included as additional codes as these were not identified within the
BCT. These additional codes were identified once each: "Perceived choice" [29] and "Self-prophecy" [28] (Table 3).

\section{Effectiveness of the BCTs}

The study reporting the intervention with the largest effect size $(d=1.45)$ used "Pros/cons" and "Problem solving" [16]. This study also reported two additional 


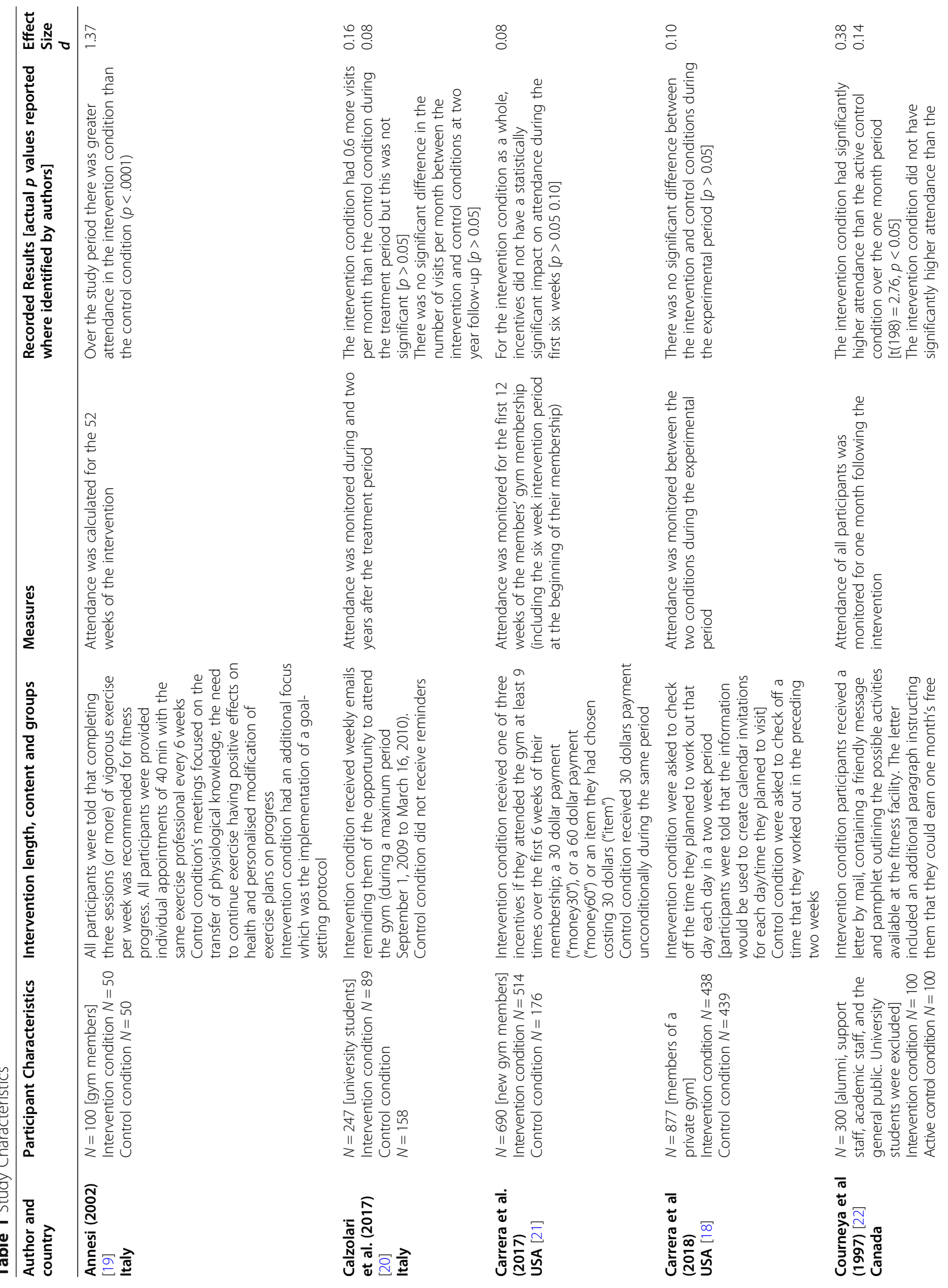




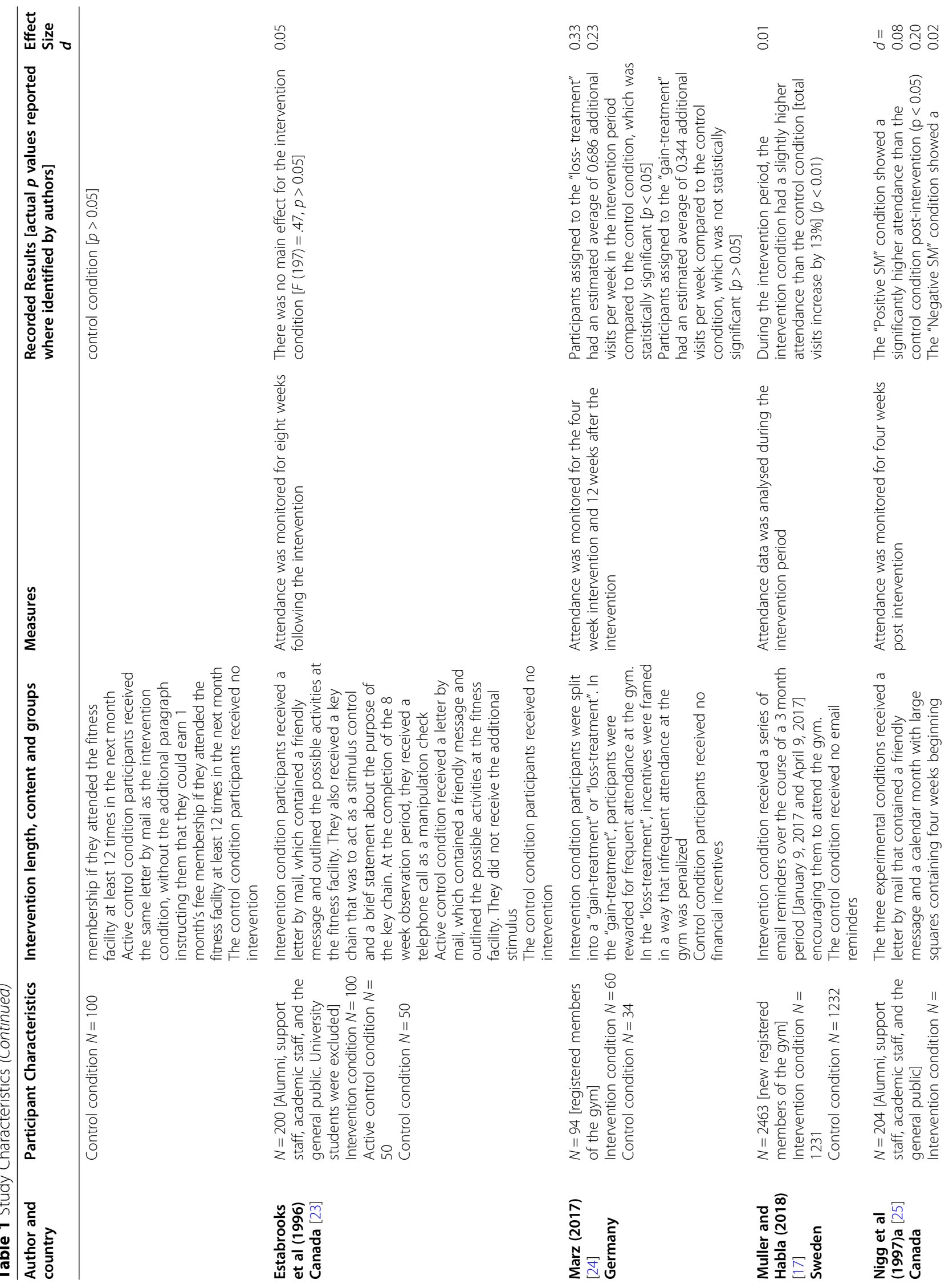




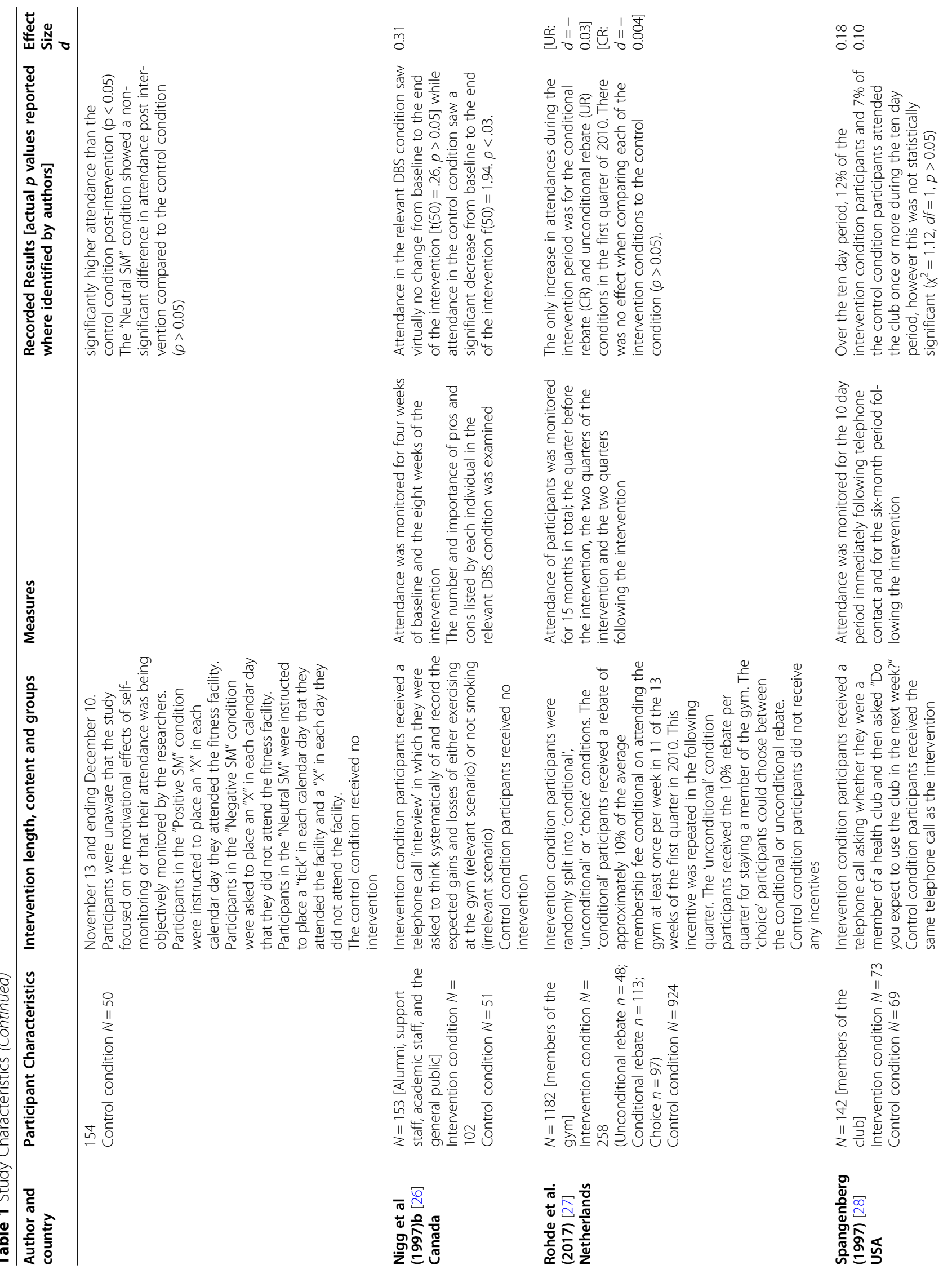




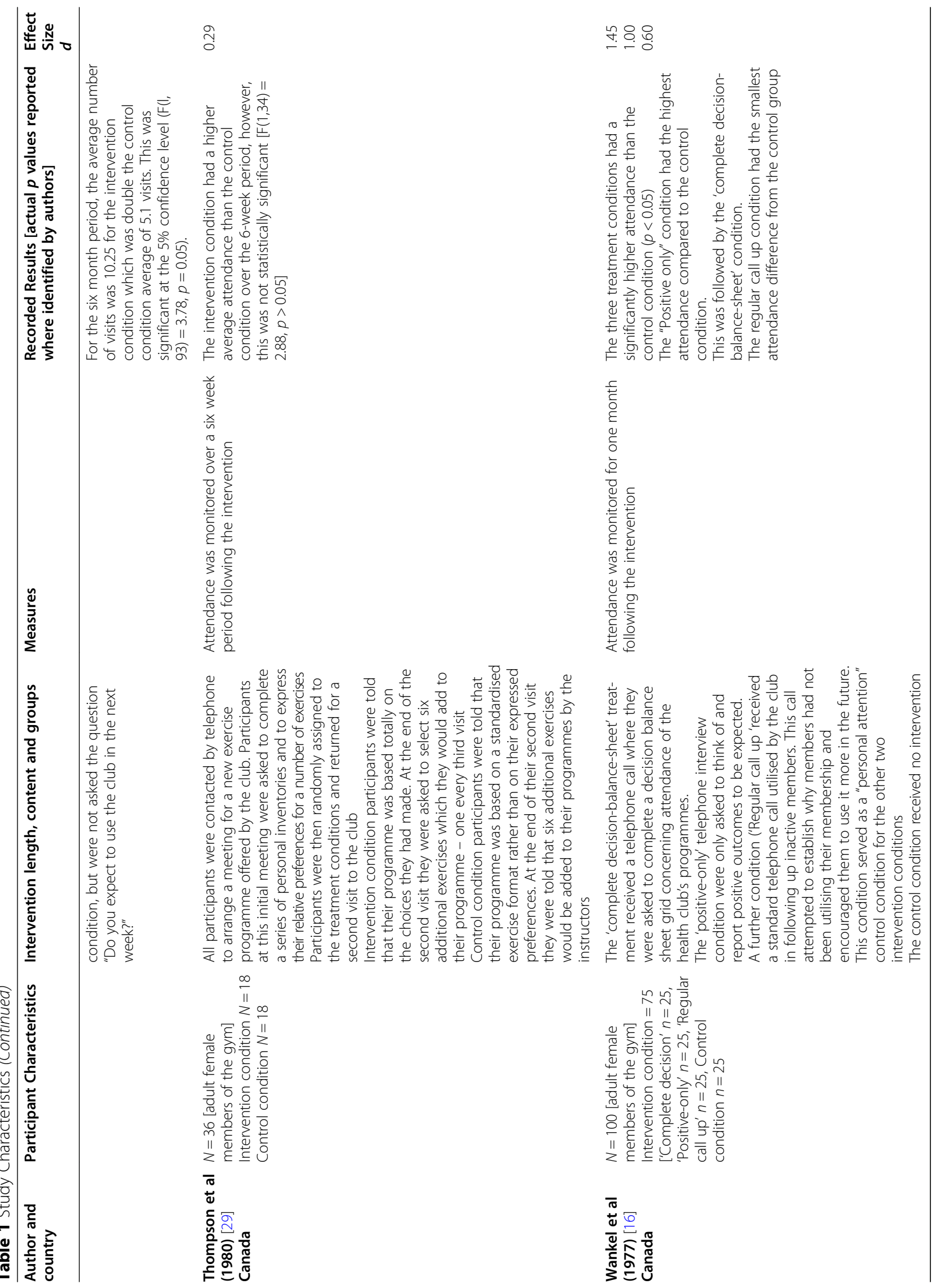


Table 2 Bias Coding for Included Studies

\begin{tabular}{|c|c|c|c|c|c|c|c|}
\hline & $\begin{array}{l}\text { Random } \\
\text { sequence } \\
\text { generation } \\
\text { (Selection Bias) }\end{array}$ & $\begin{array}{l}\text { Allocation } \\
\text { concealment } \\
\text { (Selection } \\
\text { bias) }\end{array}$ & $\begin{array}{l}\text { Blinding of } \\
\text { participants and } \\
\text { personnel } \\
\text { (Performance bias) }\end{array}$ & $\begin{array}{l}\text { Blinding of } \\
\text { outcome } \\
\text { assessment } \\
\text { (Detection bias) }\end{array}$ & $\begin{array}{l}\text { Incomplete } \\
\text { outcome data } \\
\text { (Attrition bias) }\end{array}$ & $\begin{array}{l}\text { Selective } \\
\text { reporting } \\
\text { (Reporting } \\
\text { bias) }\end{array}$ & $\begin{array}{l}\text { Other } \\
\text { sources of } \\
\text { bias (Other } \\
\text { bias) }\end{array}$ \\
\hline $\begin{array}{l}\text { Annesi (2002) } \\
\text { [19] }\end{array}$ & Low & Low & Unclear & Low & Low & Low & Low \\
\hline $\begin{array}{l}\text { Calzolari et al. } \\
\text { (2017) [20] }\end{array}$ & High & High & Unclear & Low & Low & High & Low \\
\hline $\begin{array}{l}\text { Carrera et al. } \\
\text { (2017) [21] }\end{array}$ & Low & Low & Unclear & Low & Low & Low & Low \\
\hline $\begin{array}{l}\text { Carrera et al. } \\
\text { (2018) [18] }\end{array}$ & Low & Low & Unclear & Low & Low & Low & Low \\
\hline $\begin{array}{l}\text { Courneya } \\
\text { et al. (1997) } \\
\text { [22] }\end{array}$ & Low & Low & Unclear & Low & Low & Low & Low \\
\hline $\begin{array}{l}\text { Estabrooks } \\
\text { et al. (1996) } \\
\text { [23] }\end{array}$ & Low & Low & Unclear & Low & Low & Low & Low \\
\hline $\begin{array}{l}\text { Marz (2017) } \\
{[24]}\end{array}$ & Low & Low & Unclear & Low & High & Low & Low \\
\hline $\begin{array}{l}\text { Muller and } \\
\text { Habla (2018) } \\
{[17]}\end{array}$ & Low & Low & Unclear & Low & Low & Low & Low \\
\hline $\begin{array}{l}\text { Nigg et al. } \\
\text { (1997)a [25] }\end{array}$ & Low & Low & Unclear & Low & Low & Low & Low \\
\hline $\begin{array}{l}\text { Nigg et al. } \\
\text { (1997)b [26] }\end{array}$ & Low & Low & Unclear & Low & Low & Low & Low \\
\hline $\begin{array}{l}\text { Rohde et al. } \\
\text { (2017) [27] }\end{array}$ & Low & Low & Unclear & Low & Low & Low & Low \\
\hline $\begin{array}{l}\text { Spangenberg } \\
\text { (1997) [28] }\end{array}$ & Low & Low & Unclear & Low & Low & Low & Low \\
\hline $\begin{array}{l}\text { Thompson } \\
\text { et al. (1980) } \\
\text { [29] }\end{array}$ & Low & Low & Unclear & Low & Low & Low & Low \\
\hline $\begin{array}{l}\text { Wankel et al. } \\
\text { (1977) [16] }\end{array}$ & Low & Low & Unclear & Low & Low & Low & Low \\
\hline
\end{tabular}

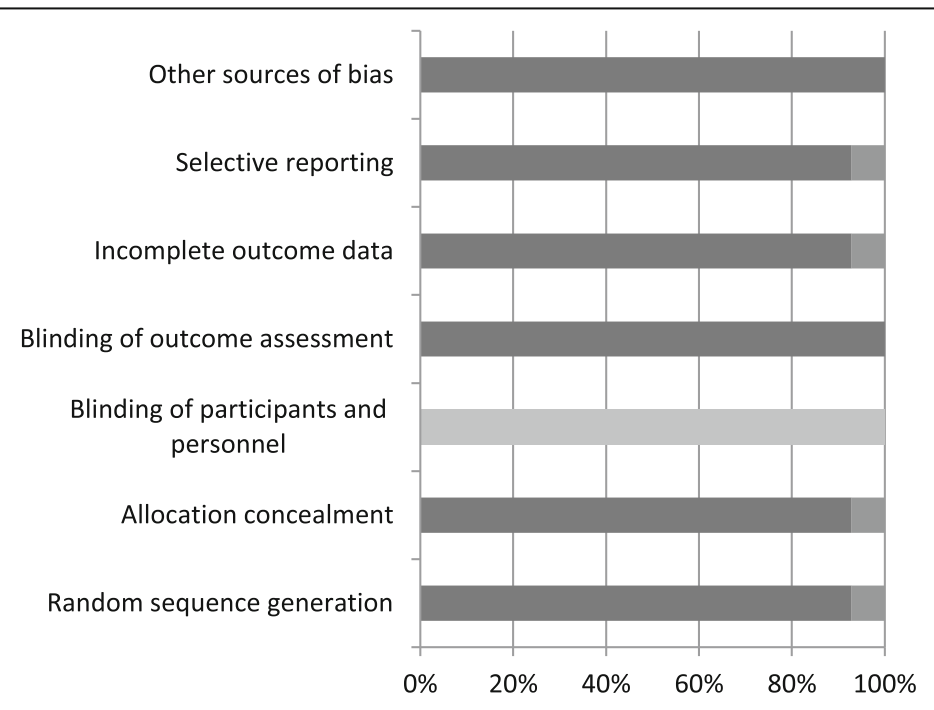

- Low risk of bias

Unclear risk of bias

High risk of bias

Fig. 2 Bias Chart for Included Studies 


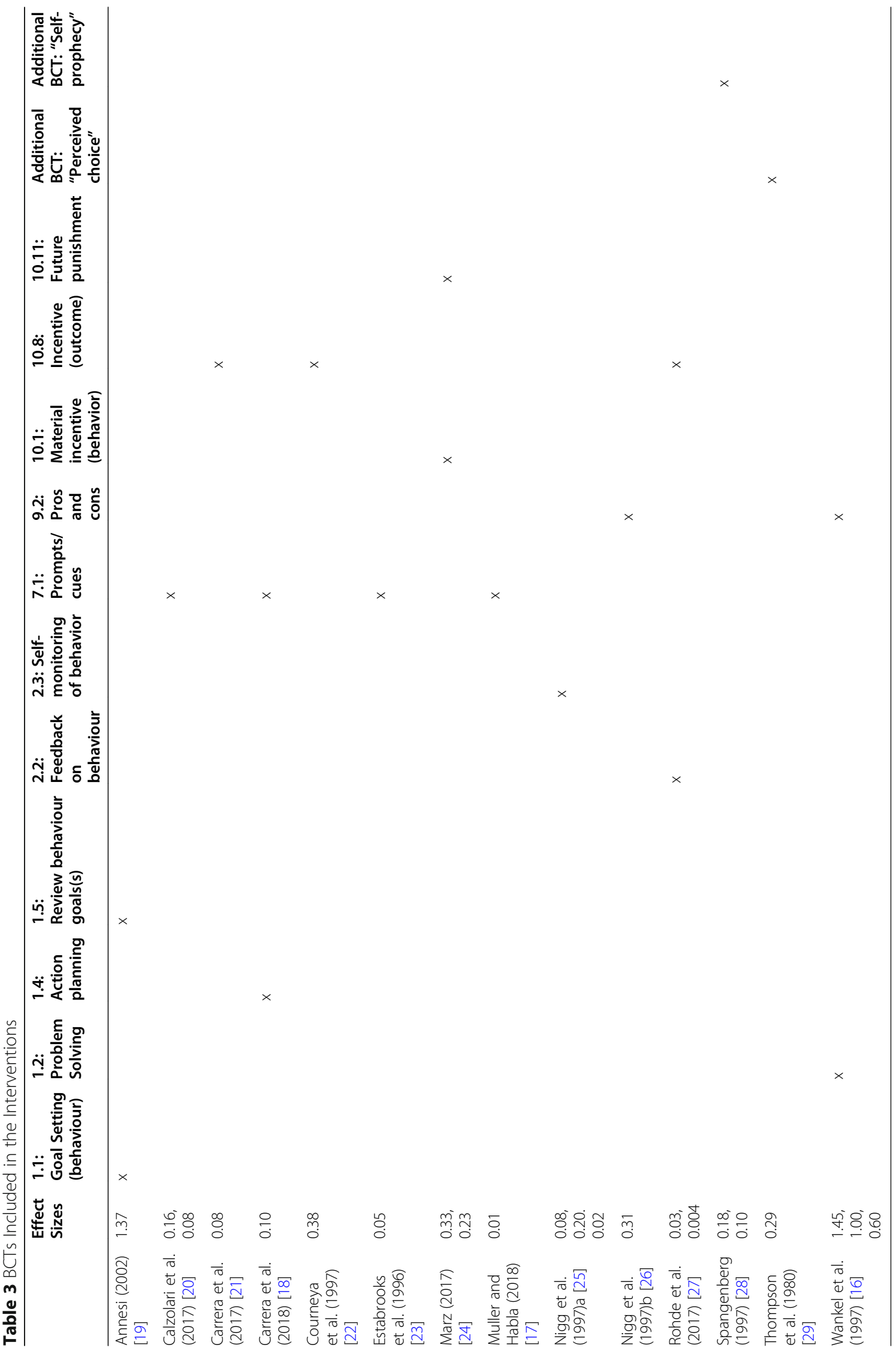


interventions using "Pros/cons" and "Problem solving"; one with a large effect size $(d=1.00)$ and one with a medium effect size $(d=0.60)$. One other intervention had a large effect size $(d=1.37$ [19]) using "Goal setting (behaviour)" and "Review behaviour goals". These BCTs were not used in any of the other interventions. The BCTs used in interventions associated with small or trivial effect sizes were as follows: "Incentive (outcome)" $(d=0.004, d=0.03$, $d=0.08, d=0.14, d=0.29, d=0.38$ ); "Material incentive (behaviour)" ( $d=0.04, d=0.23, d=0.33)$; Future punishment" ( $d=0.04, d=0.23, d=0.33)$; "Pros and cons" $(d=$ $0.31)$; "Perceived choice" $(d=0.29) ;$ "Self-prophecy" $(d=$ $0.10, d=0.18)$; "Prompts/cues" ( $d=0.01, d=0.05, d=0.06$, $d=0.10, d=0.16)$; "Action Planning" $(d=0.10)$; "Selfmonitoring of behaviour" $(d=0.08)$; and "Feedback on behaviour" ( $d=0.004, d=0.03)$.

\section{Discussion}

\section{Main findings}

The main aim of this systematic review was to understand the effectiveness of behaviour change interventions that aimed to increase attendance of members in health and fitness venues. Interventions with the largest effects on attendance utilised problem solving, pros/cons, goal setting (behaviour) and reviewing behaviour goals as behaviour change techniques (BCTs). Aside from one other intervention which had a medium effect size and also utilised problem solving and pros/cons, the remaining interventions had small or trivial effects on attendance behaviour. Given that only two studies (with combined sample size of 475) showed a moderate to large effect size, there is a limited evidence base from which to draw extensive conclusions on which BCTs could be effective in increasing attendances at health and fitness venues.

Pros/cons and problem solving showed the strongest evidence of effectiveness thereby demonstrating the potential utility of these techniques to increase attendances at health and fitness venues. The decisional balance of perceived advantages and disadvantages of change, such as pros/cons, is identified as one of three key factors that mediate behaviour change within the transtheoretical model of behaviour change (TTM) [30]. However, it should be noted that one intervention utilising pros/cons as the only BCT in the current review had a small effect $(d=0.31)$ [26]. The findings could be influenced by which BCTs pros/cons is paired with. Thus more research is therefore necessary to understand how this BCT can be most effectively applied to increase attendances at health and fitness venue.

The second highest effect sizes were found for interventions that included the BCTs goal-setting (behaviour) and review behaviour goals. These BCTs have been found to be effective techniques in a previous meta-analysis of PA interventions which found that interventions that combined goal setting along with self-monitoring [31] had the largest effect sizes. The meta-analysis also found that other behaviour change techniques derived from control theory [32], such as prompting intention, providing feedback on performance, and prompting review of goals were associated with larger effect sizes [31]. Interventions derived from control theory have also been found to be associated with greater changes in intention and stages of change in a review of how interventions can increase motivation for PA [33]. In the intervention included in the current review [19], members also met with a health and fitness professional every six weeks which suggests that face-to-face contact could be a good means through which to review behavioural goals.

The most common behaviour change technique, used in four studies, was prompts/cues [17, 18, 20, 23]. The second most common behaviour change technique, used in three studies, was financial incentives; however, the effects of financial incentives on attendance were small or trivial $[21,22,27]$, although when financial incentives were framed as a 'loss' they had a stronger effect $(d=$ 0.33 ) on attendance [24]. The behavioural economics literature has a wealth of research investigating the 'loss aversion' effect on individuals' behaviour, notably that individuals tend to prefer avoiding losses than acquiring equivalent gains [34]. The majority of this research has been related to monetary gains and losses and how individuals respond to various decisions related to how much they could gain or lose in a specific situation. Further research is needed to understand how the users of health and fitness venues respond to the framing of financial losses and rewards to incentivise attendance.

The mode of delivery might also impact on intervention effectiveness. For example, the intervention with the highest effect size [16] was delivered via telephone and the second largest effect size [19] was delivered 'face-toface' such that participants attended a number of prearranged 40 min sessions with a health and fitness professional. These methods of delivery were in contrast to many of the studies that had small or trivial effect sizes. In the two studies that had the smallest effect sizes ([22] [23];) participants had minimal face-to-face contact. For example, in both of these studies, the intervention conditions received the intervention in the post with instructions of what they needed to do. It could be that participants had low engagement with these interventions which may partially explain the trivial and nonsignificant effects. Similarly trivial effects were reported in other studies which had minimal face-to-face contact $[20,21]$. One potential advantage of using methods not requiring personal contact is the high number of participants they can reach. However, these delivery methods may have lower effectiveness due to lower levels of 
participant engagement. Cost-effectiveness studies are therefore required to explore this trade-off between scale and engagement in interventions. Given the current findings it would also appear important to understand how interventions that have minimal direct contact with participants can be effective in increasing attendances.

\section{Implications of findings}

This systematic review identified 14 studies reporting 20 interventions that sought to increase attendance in members at health and fitness venues. Of these, only three interventions showed a large effect. Given the results in the current review, interventions could include pros/cons alongside problem solving techniques and goal setting alongside reviewing behaviour goals to increase attendance in health and fitness venues. It is important to note that these findings were from only two separate studies; these implications should therefore be treated with caution. The inclusion of other BCTs taken from control theory, such as self-monitoring, should also be considered as they have been associated with large effect sizes in increasing motivation for PA [33]. There are also implications for the delivery of interventions. In particular, using a direct contact method of delivery may increase intervention effectiveness as it may lead to greater engagement than methods that do not directly interact with participants. Notably, the BCTs with the highest effect sizes were only reported in two studies. Although these could be effective in increasing attendances, additional research is required to replicate these findings. Apart from the use of four BCTs, other interventions included in the review had only small or trivial effects on attendance. More studies are needed to test a greater range of theory-based BCTs that have been found to be effective in other contexts. Identifying the $\mathrm{BCTs}$ that are best able to increase attendances at health and fitness venues may also help to increase PA at a population level given the large numbers of people who are members of such venues, but currently under-utilise them.

\section{Strengths and limitations of this review}

The current review had a number of strengths. First, it is the first systematic review to evaluate the effectiveness of interventions to increase attendance in health and fitness venues. Second, the study reviewed studies that had electronically recorded attendance at the health and fitness venue. This measurement provides an objective assessment of attendance at venues and potential change as a result of interventions. Third, the utilisation of the behaviour change taxonomy also enabled a more detailed and systematic analysis of the likely active ingredients of successful interventions.
The current review also had some limitations. First, the conclusions are based on only 14 studies. More studies are therefore needed to identify the BCTs and components of interventions that could increase attendances at health and fitness venues. Second, none of the studies explicitly described the BCTs used within the study. The studies in the current review had to be coded to identify which BCTs had been included, often on the basis of limited information. Third, the studies included different monitoring periods which might have reduced the ability to compare effectiveness between interventions. However, there was no evidence that the length of the monitoring period was associated with larger or smaller effect sizes. Finally, the behaviour change technique taxonomy did not cover all of the BCTs identified.

\section{Conclusion}

Overall, this systematic review has reported on the current evidence base on which BCTs can be effective in increasing attendance at health and fitness venues. Whilst the available evidence suggests utilising pros/cons alongside problem solving and goal-setting (behaviour) alongside reviewing behaviour goals may be effective, there are only a limited number of studies in this field. Small sample sizes and small effect sizes across the majority of interventions make it difficult to draw definitive conclusions and further studies are therefore required to provide greater certainty about which techniques BCTs are likely to increase attendances at health and fitness venues.

\section{Abbreviations}

PA: Physical activity; PHE: Public Health England

\section{Acknowledgements \\ We would like to thank the ESRC for their funding towards the research project.}

\section{Authors' contributions}

MR developed the design, conducted the analysis and wrote the manuscript. $\mathrm{PN}$ and EG reviewed and critiqued the manuscript. All authors have read and approved the manuscript.

\section{Funding}

This study was funded by the Economic and Social Research Council (ESRC). The funder had no role in the design of the study, the collection, analysis, interpretation of data, writing the manuscript or the decision to submit for publication.

\section{Availability of data and materials}

All data generated or analysed during this study are included in this published article.

Ethics approval and consent to participate Not applicable.

Consent for publication

Not applicable.

Competing interests

The other authors have no conflicts of interest. 


\section{Author details}

${ }^{1}$ School of Health and Related Research, The University of Sheffield, 30 Regent St, Sheffield S1 4DA, UK. ${ }^{2}$ Department of Psychology, The University of Sheffield, Cathedral Court, The University of Sheffield, 1 Vicar Ln, Sheffield S1 2LT, UK.

Received: 26 May 2020 Accepted: 16 November 2020

Published online: 07 December 2020

\section{References}

1. World Health Organisation (2018). Global recommendations on physical activity for health. http://www.who.int/dietphysicalactivity/factsheet_ recommendations/en/. Accessed 24 Mar 2020.

2. Public Health England: Health matters: getting every adult active every day. https://www.gov.uk/government/publications/health-matters-getting-everyadult-active-every-day/health-matters-getting-every-adult-active-every-day (2016). Accessed 24 Mar 2020.

3. Priest N, Armstrong R, Doyle J, Waters E. Interventions implemented through sporting organisations for increasing participation in sport. Cochrane Database Syst Rev. 2008;16:3.

4. Deloitte (2018). European Health and Fitness Report 2018. http://www.who.int/ dietphysicalactivity/factsheet_recommendations/en/. Accessed 24 Mar 2020

5. The European Commission (2014). Becoming the hub: the health and fitness sector and the future of health enhancing physical activity. http:// eose.eu/wp-content/uploads/2014/03/HUB_THE-FINAL-REPORT.pdf . Accessed 24 Mar 2020

6. Crossley N. In the gym: motives, meaning and moral careers. Body Soc. 2006;12:23-50

7. Rand M, Goyder E, Norman P, Womack R. Why do new members stop attending health and fitness venues? The importance of developing frequent and stable attendance behaviour. Psychol Sport Exerc. 2020; 101771.

8. DellaVigna S, Malmendier U. Paying not to go to the gym. Am Econ Rev. 2006;96:694-719.

9. World Health Organisation (2018). Interventions on Diet and Physical Activity: What Works. https://www.who.int/dietphysicalactivity/whatworks/ en/. Accessed 8 Aug 2020

10. Michie S, Richardson M, Johnston M, Abraham C, Francis J, Hardeman W, et al. The behavior change technique taxonomy (v1) of 93 hierarchically clustered techniques: building an international consensus for the reporting of behavior change interventions. Ann Behav Med. 2013:46:81-95.

11. Michie $S$, Johnston $M$. Theories and techniques of behaviour change: developing a cumulative science of behaviour change. Health Psychol Rev. 2012;6:1-6.

12. Michie S, Abraham C, Whittington C, McAteer J, Gupta S. Effective techniques in healthy eating and physical activity interventions: a metaregression. Health Psychol. 2009;28:690-701.

13. Bothwell LE, Greene JA, Podolsky SH, Jones DS, Malina D. Assessing the gold standard - lessons from the history of RCTs. N Engl J Med. 2016;374: 2175-81.

14. Cohen J. Statistical power analysis for the behavioral sciences. 2nd ed. Hillsdale NJ: Lawrence Erlbaum Associates; 1988

15. Higgins J, Altman D, Gotzsche P, Juni P, Moher D, Oxman A, et al. The Cochrane Collaboration's tool for assessing risk of bias in randomised trials. Br Med J. 2011;343:5928.

16. Wankel L, Thompson C. Motivating people to be physically active: Selfpersuasion vs balanced decision making. J Appl Soc Psychol. 1977;7:332-40.

17. Muller $P$, Habla W. Experimental and non-experimental evidence on limited attention and present Bias at the gym. ZEW Centre for Eur Econ Res. 2018; 18-041. https://madoc.bib.uni-mannheim.de/46998/1/dp18041.pdf.

18. Carrera M, Royer H, Stehr M, Sydnor J, Taubinsky D. The limits of simple implementation intentions: evidence from a field experiment on making plans to exercise. Nat Bureau Econ Res. 2018;62:95-104. https://www. sciencedirect.com/science/article/abs/pii/S01676296183019.

19. Annesi J. Goal-setting protocol in adherence to exercise by Italian adults. Percept Mot Skills. 2002;94:453-8.

20. Calzolari G, Nardotto M. Effective reminders. Manag Sci. 2017;63:2915-32.

21. Carrera M, Royer H, Stehr M, Syndor J. Can financial incentives help people trying to establish new habits? Experimental evidence with new gym members. IZA Discussion Papers. 2017;10874.
22. Courneya KS, Estabrooks PA, Nigg CR. A simple reinforcement strategy for increasing attendance at a fitness facility. Health Educ Behav. 1997;24:708-15.

23. Estabrooks P, Courneya K, Nigg C. Effect of a stimulus control intervention on attendance at a university fitness center. Behav Modif. 1996:20:202-15.

24. Marz O. Does loss aversion beat procrastination? A behavioral health intervention at the gym. ECares Working Paper. 2017;33.

25. Nigg CR, Courneya KS, Estabrooks PA. Maintaining attendance at a fitness center: An application of the decision balance sheet. Behav Med. 1997a;23: $130-7$.

26. Nigg CR, Courneya KS, Estabrooks PA. Effects of differential self-monitoring on attendance at a fitness center. Avante. 1997b;3:64-79.

27. Rohde K, Verbeke W. We like to see you in the gym - a field experiment on financial incentives for short and long term gym attendance. J Econ Behav Organ. 2017;134:388-407.

28. Spangenberg ER. Increasing health club attendance through self-prophecy. Mark Lett. 1997;8:23-31.

29. Thompson CE, Wankel LM. The effects of perceived activity choice upon frequency of exercise behavior. J Appl Soc Psychol. 1980;10:436-43.

30. Marshall S, Biddle S. The Transtheoretical model of behavior change: a meta-analysis of applications to physical activity and exercise. Ann Behav Med. 2001;23:229-46.

31. Michie S, Fixsen D, Grimshaw J, Eccles M. Specifying and reporting complex behaviour change interventions: the need for a scientific method. Implement Science. 2009:4:40.

32. Carver CS, Scheier MF. Control theory: a useful conceptual framework for personality-social, clinical and health psychology. Psychol Bull. 1982;92:111-35.

33. Knittle K, Nurmi J, Crutzen R, Hankonen N, Beattie M, Dombrowski S. How can interventions increase motivation for physical activity? A systematic review and meta-analysis. Health Psychol Rev. 2018;12:211-30.

34. Novemsky N, Kahneman D. The boundaries of loss aversion. J Mark Res. 2005:42:119-28.

\section{Publisher's Note}

Springer Nature remains neutral with regard to jurisdictional claims in published maps and institutional affiliations.

Ready to submit your research? Choose BMC and benefit from:

- fast, convenient online submission

- thorough peer review by experienced researchers in your field

- rapid publication on acceptance

- support for research data, including large and complex data types

- gold Open Access which fosters wider collaboration and increased citations

- maximum visibility for your research: over $100 \mathrm{M}$ website views per year

At BMC, research is always in progress.

Learn more biomedcentral.com/submissions 